\title{
Incidencia de reacciones adversas a la donación de sangre 2006 a 2009
}

\author{
Lucía Luna Mendoza1, Lucila Rojas Saldaña ${ }^{1}$ Lidia Cruz Rodríguez ${ }^{1}$, María Luisa Suaste Mendoza \\ Ana María Mejía Domínguez ${ }^{2}$
}

RESUMEN

La donación de sangre es un acto de humanismo que conlleva una serie de mecanismos intrínsecos y extrínsecos que la convierten en un proceso complejo, entre los que resaltan: proteger, conservar y promover la salud e integridad del donante. Objetivo: determinar frecuencia y tipo de reacciones adversas a la donación de sangre (RAD) entre los años 2006 a 2009. Metodología: estudio descriptivo, retrolectivo y longitudinal del $1^{\circ}$ de enero de 2006 al 31 de diciembre de 2009. Se revisaron historias clínicas del archivo del banco de sangre, se diseñó una base de datos en Microsoft Office Excel 2003; para la recopilación y análisis de los mismos. Resultados: de 31,176 donadores de sangre se presentaron 1,789 RAD en 767 sujetos, con una razón RAD/donante de 1,7; siendo el año 2009; donde se presentó el mayor número de eventos (31.4\%), el género en el que predominaron dichas RAD fue el masculino (53.1\%), edad promedio global osciló entre los $29 \pm 9$ años, IMC global fue $32.15 \pm$ $3.87 \mathrm{~kg} / \mathrm{m}^{2}$, nivel socioeconómico promedio bajo, de procedencia foránea. Las RAD más frecuentes fueron: mareo 91.5\% y náusea $43.2 \%$; entre los factores considerados influyentes para RAD destacan: ser donador de primera vez, tiempo de ayuno superior a 6 hs y no haber conciliado el sueño entre una y catorce hs; la incidencia global de RAD fue de $5.73 \%$ en 767 sujetos, las horas prolongadas de ayuno, edad y ser donador de primera vez fueron los factores más influyentes para los RAD.

Palabras claves: reacciones adversas, donación de sangre, donación, mareo, convulsión.

\section{Incidence of adverse reactions to blood donation 2006-2009}

\begin{abstract}
Blood donation is an act of humanism that leads to a series of intrinsic and extrinsic mechanisms that make it a complex process who emphasize protecting, conserving and promoting the health and integrity of the donor. Objective: to determine the frequency and type of adverse reactions to blood donation (RAD) between the years 2006 and 2009. Methodology: a descriptive, retrospective and longitudinal study since January $1^{\text {th }} 2006$ to December 31 2009. It was reviewed the total of donor's medical histories from the blood bank's medical file records of the file from the blood bank, It Was Designed to database in Microsoft Office Excel 2003 for the collection and analysis of them. Results: blood donors 31.1761 .789 RAD occurred in 767 subjects, with a ratio RAD / donor of 1.7, being the year 2009 where the largest number of events (31.4\%), the predominant genre in which such RAD was males (53.1\%), overall mean age ranged from $29 \pm 9$ years, overall BMI was $32.15 \pm 3.87 \mathrm{~kg} / \mathrm{m} 2$, low average socioeconomic status of foreign origin. The RAD most common were nausea, dizziness $91.5 \%$ and $43.2 \%$, among the factors considered influential in RAD are: to be a donor for the first time, fast time over 6 hours and did not sleep between 1 and 14 hours; the overall incidence of RAD was 5.73\% in 767 subjects, extended hours of fasting, age, hours of fasting and be the first time donor were the most influential factors for RAD.
\end{abstract}

Key words: adverse reactions, blood donation, donation, dizziness, seizures.

Correspondencia: Lucía Luna Mendoza. Instituto Nacional de Cardiología Dr. Ignascio Chávez. Juan Badiano \# 1, Col. Sección XVI, 14080. México, D.F. E-mail: lucyetni-@hotmail.com

$\mathrm{L}$ a donación de sangre es un acto de humanismo que conlleva una serie de mecanismos intrínsecos y extrínsecos que la convierten en un proceso complejo, en el cual se deben considerarse varios aspectos, entre ellas se destaca: proteger, conservar y promover la salud e integridad del donante. Como se menciona en el artículo octavo de la Declaración Universal de Bioética y Derechos Humanos de la UNESCO (2005) que a la letra dice, al aplicar y fomentar el 
conocimiento científico, debe tomar en cuenta la vulnerabilidad; de los individuos y grupos en especial frágiles los cuales deben ser protegidos; así como, respetar su integridad personal.

En este sentido, el profesional de enfermería del banco de sangre al formar parte de un cuerpo medular multidisciplinarío, y tener relación directa con individuos sanos, no la exime de aplicar los principios de respeto, cuidado de la salud e integridad de las personas, por lo que protegerlas de cualquier daño es un principio fundamental; por lo tanto se debe propiciar un entorno seguro donde se otorguen cuidados de enfermería de calidad y libres de todo riesgo, sin perder de vista que fomentar la cultura del autocuidado a través de la información y enseñanza con un enfoque de prevención, favorecen la participación e interacción del hombre dentro y fuera de su comunidad.

Conocer la incidencia de reacciones adversas a la donación de sangre (RAD), que se presentan en el centro, permite tener un panorama amplio sobre el comportamiento de la población con la que se trabaja de manera cotidiana facilitando, así la estructura de un programa de enfermería que cuente con herramientas necesarias que permitan una donación efectiva libre de riesgo como parte del proceso de hemovigilancia.

\section{MATERIAL Y MÉTODOS}

Se realizó un estudio descriptivo, retrospectivo y longitudinal del $1^{\circ}$ de enero de 2006 al 31 de diciembre de 2009. La población estuvo constituida por el total de donadores alogénicos de ambos sexos que acudieron al banco de sangre del Instituto Nacional de Cardiología Ignacio Chávez durante este periodo y que cumplieron con los criterios establecidos por la NOM003-SSA2-1993 ${ }^{1}$ para la disposición de sangre humana y sus componentes con fines terapéuticos; se incluyeron a todos los donadores que presentaron RAD de forma no aleatorizada; no hubo criterios de exclusión ni de eliminación, quedando constituida la muestra por el total de donadores con RAD. La información se extrajo de historias clínicas del archivo del banco de sangre.

Los datos obtenidos fueron procesados en una base de datos en Microsoft Office Excel 2003.

Las variables independientes fueron: sexo, edad, peso, talla, índice de masa corporal (IMC), presión arterial, horas efectivas de sueño, horas reales de ayuno, número de donaciones previas; la variable dependiente fue el tipo de RAD; se analizó la información mediante medidas de tendencia central.

\section{RESULTADOS}

De 31,176 donadores de sangre alogénicos entre los años 2006 y 2009 de ambos sexos, hubo 1,789 RAD constituyendo la muestra que representa el 5.73\% de la población. Del total de RAD; $17.2 \%$ se presentaron en el 2006, 20.9\% en el 2007, 30.5\% en 2008 y 31.4\% en 2009 (cuadro 1).

Cuadro 1. Frecuencia de reacción adversas a la donación de sangre por año.

\begin{tabular}{lccc}
\hline Año & $\begin{array}{c}\mathrm{N} \\
\text { (donaciones por año) }\end{array}$ & $\begin{array}{c}\text { Frecuencia de } \\
\text { RAD }\end{array}$ & $(\%)$ \\
\hline 2006 & 7,600 & 132 & $(17.2)$ \\
2007 & 8,060 & 160 & $(20.9)$ \\
2007 & 8,370 & 234 & $(30.5)$ \\
Total & 31,176 & 767 & $(100)$ \\
\hline
\end{tabular}

Fuente: Cédulas de valoración de RAD aplicadas en el Banco de Sangre del INCICh.

Estas RAD se presentaron en 767 (2.46\%) donantes, con una razón $\mathrm{RAD}$ /donante de 1,7 con un rango de 1,10 RAD por persona. Las RAD se presentaron con mayor frecuencia en hombres (53.1\%).

La edad osciló entre los $29 \pm 9$ años, el peso promedio registrado fue $71 \pm 12.5 \mathrm{~kg}$. La talla en general se observó en $163 \pm 9.3 \mathrm{~cm}$. El IMC global fue $32.15 \pm 3.87 \mathrm{~kg} / \mathrm{m}^{2}$. El nivel socioeconómico promedio fue bajo y la procedencia foránea. La frecuencia porcentual de RAD se distribuyó de la siguiente forma: mareo 91.5\%, náusea $43.2 \%$, palidez $28.7 \%$, diaforésis $21.3 \%$, lipotimia $14.7 \%$, vómito $10.6 \%$, convulsión $8.1 \%$, hormigueo $5.5 \%$, escalofrío $4.6 \%$, disnea $3.5 \%$ y relajación de esfínteres con $1.7 \%$ (figura 1). La frecuencia anual de RAD por género se describe en el cuadro 2.

Cuadro 2. Frecuencia anual de reacciones adveras por género.

\begin{tabular}{|c|c|c|c|c|c|}
\hline \multirow{3}{*}{$\begin{array}{l}\text { AÑO } \\
2006\end{array}$} & \multicolumn{3}{|c|}{ Género } & \multicolumn{2}{|c|}{ Total (\%) } \\
\hline & \multirow{2}{*}{$\begin{array}{c}\text { Femenino } \\
58(4.39\end{array}$} & \multicolumn{2}{|c|}{ Masculino } & & \\
\hline & & 74 & $(56.1)$ & 132 & (17.2) \\
\hline 2007 & $72(45.0)$ & 88 & $(55.0)$ & 160 & (20.9) \\
\hline 2008 & $113(48.3)$ & 121 & (51.7) & 234 & (30.5) \\
\hline 2009 & $117(48.1)$ & 124 & $(51.9)$ & 241 & (31.4) \\
\hline Total & $360(46.9)$ & 407 & $(53.1)$ & 767 & (100) \\
\hline
\end{tabular}

Fuente: cédulas de valoración de RAD aplicadas en el Banco de Sangre del INCICh.

Los factores relacionados con las 1,789 RAD reportadas, con más frecuencia registradas son: ayuno con un rango de 4 a 23 hs; se presentaron casos en los que el tiempo de no haber conciliado el sueño fluctúo entre 1 y 14 hs la presión arterial sistólica mantuvo una media de $107 \pm 11 \mathrm{mmHg}$; la presión arterial diastólica se mantuvo con una media de $67 \pm 8.5 \mathrm{mmHg}$, cifras que no reflejan alteración que se puedan considerar como factor de riesgo para RAD (cuadro 3), en los sujetos cuya donación era la primera se obtuvo un 69.7\% distribuidos de la siguiente forma: 2006; 83 (10.8\%) sujetos, 2007; 117 (15.2\%), 2008; 154 (20.0\%) y 2009; 181 (23.5 \%); el resto de los factores documentados no se consideran relevantes para RAD. 


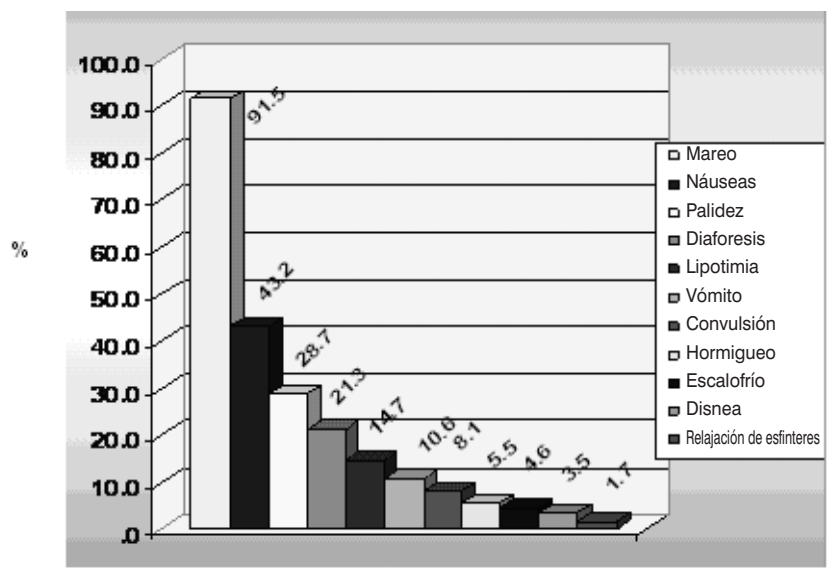

Reacciones adversas a la donación

Fuente: cédulas de valoración de RAD aplicadas en el Banco de Sangre del INCICh.

Figura 1. Tipo de reacciones adversas a la donación de sangre 20062009 .

Cuadro 3. Variables estudiadas como factores predictores de RAD.

\begin{tabular}{lcccccccc}
\hline \multicolumn{1}{c}{$\begin{array}{l}\text { Variable } \\
\text { Std }\end{array}$} & \multicolumn{2}{c}{2006} & \multicolumn{2}{c}{2007} & \multicolumn{2}{c}{ Año } & \multicolumn{2}{c}{2008} \\
& Media & Des. std & Media & Des. std & Media & Des. std & Media & Des. std \\
\hline Edad & 30.6 & 9.1 & 28.9 & 8.6 & 29.4 & 8.8 & 29.3 & 9.2 \\
Peso & 72.9 & 14.6 & 69.4 & 11.4 & 70.7 & 12.1 & 70.5 & 12.6 \\
Talla & 164 & 9.3 & 159 & 9 & 163 & 9 & 163 & 9 \\
Horas de ayuno & 5.6 & 4.3 & 9.2 & 4.4 & 13.2 & 3 & 13.4 & 2.4 \\
Horas de sueño & 5.9 & .22 & 6 & .76 & 6 & 1.68 & 6.2 & 1.64 \\
PAD (mmHg) & 70 & 8.5 & 66 & 6.6 & 67 & 8.2 & 67 & 7.6 \\
PAS (mmHg) & 112 & 12.9 & 107 & 11.4 & 108 & 11.4 & 107 & 11.1 \\
\hline Fuente: cédulas de valoración de RAD aplicadas en el Banco de Sangre del INCICh.
\end{tabular}

\section{DISCUSIÓN}

Se observó que el sexo más vulnerable a dichos eventos fue la población masculina, quizás la incidencia mayor de RAD en este género se deba a ello, coincidiendo con lo publicado por Aguirre-Gómez en un estudio realizado en 2001, donde reporta que de 14,798 donaciones, la frecuencia de RAD fue de 25 casos y de ellos 13 se presentaron en hombres².

Contrario a lo que menciona María Munera y Blanca S. Ramírez en un estudio publicado en 2001; donde consideran al sexo femenino como un factor predispuesto a reacciones vagales quizá secundaria al mayor volumen de sangre pérdido en donantes de bajo peso y estatura ${ }^{3}$.

En relación a las variables sociodemográficas, llama la atención la edad y experiencia de los sujetos en el acto de donar, considerar que la edad temprana y donación por primera vez es un factor de riesgo para presentar $\mathrm{RAD}$, como lo documenta
Vidal J, Contreras, quien encontró que la edad menor a 20 años es un factor predictivo para presentar una RAD, asociado a un peso promedio de $55 \mathrm{~kg}$; de la misma forma Rojas Saldaña en 2007; registra 63 sujetos cuya edad fluctúo entre18 y 28 de edad, con un peso promedio de 50 y $60 \mathrm{~kg}$ y 83 de los 132 casos con RAD eran donantes de primera vez $z^{4,5}$.

Varios estudios han mostrado una mayor frecuencia de reacciones adversas a la donación en donadores jóvenes. En un estudio multicéntrico sobre reacciones vasovágales en 1890; en donadores que presentaron síncope, se encontró que las variables más frecuentes son: edad, peso y donación por primera vez, esta situación se explica por que usualmente la primera donación se efectúa a temprana edad; además hay individuos jóvenes que tienen mayor sensibilidad barorreceptora ventricular más baja que los individuos mayores quienes suelen ser hemodinámicamente más estables². En lo que respecta al índice de masa corporal (IMC) no se comprobó asociación alguna con la presencia de RAD en la muestra seleccionada.

Sin embargo, se encontró que los donadores con RAD reportaban mayores horas de ayuno; asociado a esto, se observó que la mayoría de ellos presentaban un grado de estrés fuera de control, el cual no pudo ser calificado, por lo que se utilizó la observación como método para evaluar datos clínicos que sugirieran el estrés; se considera que en las condiciones antes descritas un estado de hipoglicemia puede contribuir directamente en la presencia de RAD; asimismo, miedo y nerviosismo se suman como factores predictivos de una RAD, favorecidos por la deficiente información otorgada antes de donar. El ayuno prolongado constituyó uno de los factores externos que alteran la homeostasis de personas y condicionan cambios fisiológicas en los que se ven involucrados todos los órganos blancos de la economía del sujeto implicado haciéndolo experimentar consecuentemente, situaciones de peligro cuando no las hay, generando síntomas como taquicardia, temblores, diaforésis y falta de concentración, como se menciona en la literatura ${ }^{6}$.

Otros factores asociados al ayuno prolongado no fueron analizados en esta muestra y nos obliga a evaluarlos en una futura investigación.

A pesar de que el mareo y náusea predominaron, la lipotimia y convulsión son factores; que ponen en riesgo la vida e integridad del donador. Por esta razón durante el desarrollo de la investigación se implementaron una serie de medidas preventivas en las que se enfatizó la valoración clínica de enfermería mediante la observación continua, con el fin de evaluar cambios en el grado de atención durante la entrevista y cambios en la coloración de piel durante la donación a fin de detectar a tiempo y evitar una RAD. De esta manera, Virginia Henderson describe que la persona para protegerse contra las agresiones 
externas e internas y lograr mantener su integridad física y mental, necesita ejercer un control sobre sí mismo y su entorno; aplicando medidas preventivas tales como alimentación sana, régimen de vida adecuado y utilización apropiada de determinados mecanismos de adaptación los cuales forman una red de soporte para el individuo 7 .

Por otra parte, considerando como lo plantea la misma Henderson; en la planificación de los cuidados enfermeros, en la persona cuya motivación se ve alterada, ocasiona problemas de atención y concentración, pues la angustia es catalogada como mecanismo de defensa que el donador experimenta para evitarse un daño, sobretodo cuando su cerebro interpreta peligro, ocasionando miedo, dolor anticipado y problemas para procesar lo comunicado, por tanto, la falta de atención se convierte en un factor predictivo a una $\mathrm{RAD}^{7,8}$. Por ello es de vital importancia difundir de manera coherente la información que disminuya la percepción de dichos peligros y permita tener un panorama general del proceso de la donación.

Para ello se propone el diseño e implementación de una cédula donde se lleve a cabo el registro de las RAD; asimismo, se de seguimiento al donador afectado.

Del análisis de los datos de las RAD contrastados con la literatura, quizás la hipoglicemia sea un factor predisponente a las RAD; por ello se sugiere adoptar en este centro de donación la toma de glicemia capilar predonación a toda persona, y una segunda vez a quien presente RAD, así como implementar medidas tendientes a que el donador aceptado mantenga glicemias normales predonación, como ingesta de bebidas azucaradas por lo menos 30 min antes de la donación, con el fin de aminorar la presencia de hipoglicemia y eliminar un posible factor predispuesto de RAD.

Además a la luz de los antecedentes en esta investigación y con la experiencia obtenida, se sugiere definir en el centro las etapas de donación de sangre y derivados, con el propósito de realizar una valoración clínica de las condiciones generales del donador, garantizar su estabilidad e implementar un proceso en el que se incluya la elaboración de una guía clínica de atención y un programa de educación para la salud que contenga temas dirigidos a los donadores de sangre, apoyado por el diseño de trípticos, en los que se dé a conocer de manera clara y precisa en qué consiste el proceso de donación de sangre y plaquetas, así como contar con un programa de educación continua de banco de sangre dirigido a las enfermeras, para que estas constituyan la base de una pirámide, donde el donador encuentre orientación y educación para conservar su salud, y por lo tanto disminuir al mínimo las RAD, siguiendo los preceptos de Virginia Henderson en la planificación de los cuidados enfermeros: todo ser humano está en constante necesidad de aprender y el individuo que quiere permanecer sano, combatirá la enfermedad o modificará sus hábitos deben de recibir información a menudo, para lo cual la enfermera debe valorar la integridad de las estructuras biológicas y el estado emocional del donador poniendo especial atención en aquellos individuos en los que se detecte alteración en la motivación, concentración y comunicación 7 .

A lo largo de la investigación se han implementado medidas que se mantienen en constante evaluación para conocer su efectividad, pues las RAD continúan presentándose, por lo que se considera replantear la valoración donde haya unificación en los criterios de valoración clínica del equipo multidisciplinario de salud que participa en el proceso de donación. Tomando en cuenta que los efectos psicológicos negativos que condiciona una RAD pueden resultar negativos e influir de la misma manera para futuras donaciones, por lo que, la unificación de criterios de valoración clínica favorecerá una donación inocua y permitirá disponer de componentes sanguíneos de buena calidad ${ }^{3-9}$.

\section{CONCLUSIÓN}

El proceso de donación de sangre es complejo, en el se pueden generar riesgos o daños graves a la salud e integridad del donador; por lo que el profesional de enfermería debe detectar precozmente dichos riesgos y brindar con responsabilidad cuidados de calidad que minimicen los peligros potenciales preservando de esta manera la salud e integridad de las personas. Por lo que definir las etapas del proceso de donación facilitará la detección de las áreas de mayor riesgo y permitira implementar estratégias con el fin de evitar incidentes. Por otra parte, se debe contar con un instrumento donde se registren eventos y acciones que se emplean para dar solución a la RAD. La elaboración de programas de educación continua sobre aspectos relacionados con la donación enfocados a donadores y profesionales implicados en este proceso, permitirá difundir la información de manera sistematizada y de este modo lograr la estandarización del proceso de donación como estrategia fundamental para prevención de RAD.

\section{REFERENCIAS}

1. Norma oficial Mexicana NOM-003-SSA2-1993 para la disposición de sangre humana y sus componentes con fines terapéuticos. Secretaría de Salud. 1993. [citado 24 de mayo de 2010] Disponible en: http://www.facmed.unam.mx/sss/nom/normas\%20oficiales.htm.

2. Aguirre G. Factores de riesgo para desarrollar reacción vagal severa en donadores pos-sangría. Rev Hemat 2001; 2(3):98-102.

3. Munera IM, Ramírez SB. Reacciones adversas inmediatas a la donación: frecuencia y caracterización, banco de sangre de la clínica cardiovascular Santa María Medellín 1999. Biomédica 2001;21 (3):224-7.

4. Vidal J, Contreras E, Elies F. Reacciones adversas a la donación de sangre. Boletín de la Sociedad Española de Transfusión Sanguinea. [acceso17 de mayo de 2008]; Disponible en: http://www.sets.es/ boletin-45/45-ariculos.htm. 
5. Rojas SL, Luna ML. Reacciones adversas a la donación de sangre. Rev Mex Enfer Cardiol Mayo-Agosto 2007;15(2):42-6.

6. San Péiro P, Ortíz LM. Fisiología y bioquímica en el ayuno. Rev Med Nat 2007;(1):10-19.

7. Lusis MT. Fernández FC. De la teoría a la práctica: el pensamiento de Virginia Henderson en el siglo XXI.3 ${ }^{\text {a }}$ ed. Barcelona. Masson. 2005.

8. Marina JA. Diccionario de los sentimientos. 4ta ed. España. Anagrama. 2007.

9. Radillo González A. Medicina transfusional.2a ed. México. Prado. 2006. 